\title{
Cyclizine dependence in patients with complex nutritional requirements
}

\author{
F. W. Thursby-Pelham ${ }^{1}$, A. N. De Silva ${ }^{2}$, M. A. Stroud ${ }^{2}$ and D. R. Fine ${ }^{2}$ \\ ${ }^{1}$ Department of Gastroenterology, Basingstoke \& North Hampshire NHS Foundation Trust, Basingstoke, RG24 9NA, \\ and ${ }^{2}$ Department of Gastroenterology, Southampton University Hospital NHS Trust, Southampton, SO16 6YD
}

Cyclizine is a common anti-emetic drug used throughout the UK. Misuse of cyclizine has been reported for over 40 years but these problems are not widely appreciated.

Most reports of cyclizine dependence are found in individuals suffering from traditional forms of recreational drug abuse, but it is documented in patients with chronic pain of unknown origin on long-term opiates. A case series is presented demonstrating cyclizine dependence in patients with complex nutritional problems. The aim is to highlight potential problems associated with long-term cyclizine use.

Patients with evidence of cyclizine dependence at Southampton General Hospital between June 2006 and July 2007 were identified by clinical staff. Their case notes were reviewed for historical evidence of potential cyclizine misuse.

Four patients were identified who clearly demonstrated features of cyclizine dependence. Their ages were 22, 25, 50 and 52 years and three were female. All patients had chronic abdominal pain and had complex nutritional problems. Three of the four patients had diagnoses of chronic idiopathic gut dysmotility and one (male) had short bowel syndrome secondary to a mesenteric infarct. Three of the patients required long-term parenteral nutrition whilst the other patient had prolonged nasogastric tube feeding. Two of the patients had been prescribed long-term intravenous opiates and cyclizine for pain and nausea for over 10 years whilst the other two had problems for $>1$ year.

Retrospective review of their medical notes highlighted repeated admissions where they exhibited behaviour consistent with cyclizine dependence. These features included reporting of increased pain and nausea on any attempt to withdraw cyclizine. Patients also demonstrated drug-seeking behaviour, such as: (1) stating that the only possible solution to their nausea was cyclizine; (2) describing symptoms that markedly deviated from objective clinical evidence or physical examination findings; (3) claiming that non-addictive medications 'don't work' or cannot be taken because of an allergy to them: (4) giving bizarre reasons why they had run out of cyclizine or had lost prescriptions for it; (5) manipulating situations by pitting the opinion of one doctor against that of another.

The potential problems of addiction and abuse with drugs such as benzodiazepines and opiates are well known. The authors wish to highlight that these problems can also occur with the injudicious use of cyclizine. Furthermore, these problems are not confined to patients with histories of recreational drug misuse. Although recognising cyclizine abuse is important, it would be better to prevent new cases from occurring in the first place. Therefore, with particular regard to patients suffering from chronic idiopathic pain and nausea requiring nutrition support, it is felt that cyclizine prescription should be a considered decision. 